\title{
An initiator codon mutation in SDE2 causes recessive embryonic lethality in Holstein cattle
}

\author{
Sébastien Fritz, ${ }^{\star} \dagger$ Chris Hoze, ${ }^{*} \dagger$ Emmanuelle Rebours, ${ }^{*}$ Anne Barbat, ${ }^{*}$ Méline Bizard, ${ }^{*}$ \\ Amanda Chamberlain, $\ddagger$ Clémentine Escouflaire, ${ }^{*} \dagger$ Christy Vander Jagt,‡ Mekki Boussaha, ${ }^{*}$ Cécile Grohs, ${ }^{*}$ \\ Aurélie Allais-Bonnet, †§ Maëlle Philippe,\# Amélie Vallée,Il Yves Amigues,ף Benjamin J. Hayes, $\ddagger^{* *}$ \\ Didier Boichard, ${ }^{*}$ and Aurélien Capitan ${ }^{*}{ }^{1}$ \\ *UMR GABI, INRA, AgroParisTech, Université Paris Saclay, 78350 Jouy-en-Josas, France \\ †Allice, 75595 Paris, France \\ $\ddagger$ Agriculture Victoria, AgriBio, Centre for AgriBiosciences, Bundoora 3083, Australia \\ §UMR BDR, INRA, ENVA, Université Paris Saclay, 78350 Jouy-en-Josas, France \\ \#Evolution, 35700 Rennes, France \\ \|Gènes Diffusion, 59500 Douai, France \\ ILABOGENA DNA, 78350 Jouy-en-Josas, France \\ ${ }^{* *}$ Queensland Alliance for Agriculture and Food Innovation, Centre for Animal Science, The University of Queensland, Brisbane 4067, Australia
}

\begin{abstract}
Researching depletions in homozygous genotypes for specific haplotypes among the large cohorts of animals genotyped for genomic selection is a very efficient strategy to map recessive lethal mutations. In this study, by analyzing real or imputed Illumina BovineSNP50 (Illumina Inc., San Diego, CA) genotypes from more than 250,000 Holstein animals, we identified a new locus called HH6 showing significant negative effects on conception rate and nonreturn rate at $56 \mathrm{~d}$ in at-risk versus control mating. We fine-mapped this locus in a $1.1-\mathrm{Mb}$ interval and analyzed genome sequence data from 12 carrier and 284 noncarrier Holstein bulls. We report the identification of a strong candidate mutation in the gene encoding SDE2 telomere maintenance homolog (SDE2), a protein essential for genomic stability in eukaryotes. This A-to-G transition changes the initiator ATG (methionine) codon to ACG because the gene is transcribed on the reverse strand. Using RNA sequencing and quantitative reverse-transcription PCR, we demonstrated that this mutation does not significantly affect SDE2 splicing and expression level in heterozygous carriers compared with control animals. Initiation of translation at the closest in-frame methionine codon would truncate the SDE2 precursor by 83 amino acids, including the cleavage site necessary for its activation. Finally, no homozygote for the
\end{abstract}

Received November 9, 2017.

Accepted March 5, 2018.

${ }^{1}$ Corresponding author: aurelien.capitan@inra.fr
$\mathrm{G}$ allele was observed in a large population of nearly 29,000 individuals genotyped for the mutation. The low frequency $(1.3 \%)$ of the derived allele in the French population and the availability of a diagnostic test on the Illumina EuroG10K SNP chip routinely used for genomic evaluation will enable rapid and efficient selection against this deleterious mutation.

Key words: embryonic lethality, deficit in homozygote, large-scale genotyping, SDE2

\section{INTRODUCTION}

Recent advances in genomic technologies have enabled the development of genotype-driven approaches to monitor recessive genetic defects in cattle populations. One such approach consists of exploiting large cohorts of animals that have been genotyped for genomic selection with genome-wide SNP arrays, to detect depletions in homozygous genotypes for specific haplotypes assumed to be associated with recessive deleterious mutations (e.g., VanRaden et al., 2011). Exploiting homozygous haplotype deficiency (HHD) is particularly effective in mapping mutations causing embryonic lethality or nonspecific symptoms. Indeed, this approach, supplemented by follow-up analyses, has led to the identification of 11 recessive mutations in 6 breeds: AH1 in Ayrshire (Venhoranta et al., 2014); BH2 in Brown Swiss (Schwarzenbacher et al., 2016); FH2 and FH4 in Fleckvieh (Pausch et al., 2015); HH1, HH3, HH4, and HH5 in Holstein (Fritz et al., 2013; McClure et al., 2014; Daetwyler et al. 2014; Adams et al., 2016; Schütz et al., 2016); MH1 and MH2 in Montbéliarde (Fritz et al., 2013; Michot et al., 2017); and JH1 in Jersey (Sonstegard et al., 2013). Among these, 
8 mutations were embryonic lethal and 3 (AH1, BH2, FH2) caused reduced growth and increased mortality of calves.

The power of the method depends on (1) the linkage disequilibrium between the mutation and the haplotype to be detected, (2) the penetrance of the condition in homozygous mutants, (3) the severity of this condition (i.e., the possibility for an animal to be genotyped as a candidate for genomic selection before his death or before the expression of the symptoms), (4) the amount of medium- to high-density SNP arrays available in a breed, and (5) the frequency of the haplotype or mutation within that population. Typically, the studies led so far analyzed genotyping data from 2,000 to 50,000 animals and detected deleterious haplotypes with frequencies above $2.5 \%$ in the most informative situations and above $10 \%$ for the smallest cohorts.

In this follow-up study, we mapped a new embryonic lethal haplotype segregating at low frequency in Holstein cattle by analyzing genotypes from more than 250,000 animals, and we performed complementary analyses to identify the causative mutation and characterize its effect.

\section{MATERIALS AND METHODS}

\section{Whole-Genome Screening for HHD}

Genotypes from 339,482 Holstein cattle analyzed from 2008 to 2017 with different Illumina SNP chips (BovineSNP50 Beadchip v1 and v2, Matukumalli et al., 2009; LD chip, Boichard et al., 2012a; BovineHD chip, Illumina, 2015; and EuroG10K; Illumina Inc., San Diego, CA) were extracted from the French genomic evaluation database. Pedigree information for at least 2 generations was available for the entire cohort. In total, 15,852 animals had no genotyped parents, 69,555 had only their sire genotyped, 123,229 had both their sire and dam genotyped, and 130,846 had their sire and maternal grandsire (but not their dam) genotyped. Genotypes were checked for quality and processed in the framework of the French genomic selection pipeline (Boichard et al., 2012b), including imputation and phasing with FImpute version 2.2 (Sargolzaei et al., 2014). Then, phased genotype data for 43,582 markers and 254,075 animals having either their sire and dam or their sire and maternal grandsire genotyped were analyzed using sliding windows of 20 markers (i.e., approximately $1.5 \mathrm{Mb}$ ). For each window, haplotypes were listed, their frequencies in the maternal chromosomes were estimated by counting, and 9,152,229 haplotypes with a frequency $>0.1 \%$ were considered thereafter. The number of observed homozygous progeny, $\mathbf{N}_{\mathrm{obs}}(k)$, was counted for each haplotype $k$ and compared with its expectation, $\mathbf{N}_{\exp }(k)$, under the null hypothesis of neutrality. This expectation was either estimated directly from the genotypes of the sire and the dam, or, when the genotype of the dam was not available, from the genotypes of the sire and maternal grandsire, and the frequency in the population, as described in Fritz et al. (2013). Neighbor windows with the same minimum number of homozygous products were merged to define intervals. A $\chi^{2}$ test was used to assess the significance of the difference between observed and expected results. A Bonferroni correction, consisting of multiplying the $P$-value obtained for each test by the number of haplotypes tested, was applied to account for multiple testing. A recessive defect causing embryonic or early postnatal lethality was suspected when the $\mathrm{N}_{\text {obs }}(k)$ / $\mathrm{N}_{\text {exp }}(k)$ ratio was $<0.25$ and the adjusted $P$-value $<0.01$.

\section{Haplotype Tests for HH6 and HH6s}

Phased 50k genotype data from the French genomic evaluation database were screened for 2 new haplotypes, HH6a and HH6b, on chromosome 16, showing a significant depletion in homozygous genotypes (see Results). The HH6a haplotype consists of 25 consecutive markers from ARS-BFGL-NGS-85727 (position 27,833,776 bp) to ARS-BFGL-NGS-112449 (position 29,661,958 bp); HH6b consists of 21 consecutive markers from Hapmap50900-BTA-16300 (position 31,162,715 bp) to ARSBFGL-NGS-30325 (position 32,019,139 bp; https:// support.illumina.com/downloads/bovinesnp 50v2 .html). Chromosomes carrying both HH6a and HH6b were considered to be carrying the HH6 embryonic lethal mutation.

Following the fine-mapping of the HH6 locus (see below), a second haplotype test was performed to detect a segment of 19 consecutive markers, denoted the HH6s haplotype (from Hapmap38469-BTA-12042 to ARSBFGL-NGS-87853; position $29,277,142$ to $30,262,349$ $\mathrm{bp})$. However, this haplotype test was found to be less reliable than the previous one to determine the genotypes for the HH6-associated embryonic lethal mutation because 115 animals homozygous for HH6s were observed in our panel of 254,075 individuals.

We adopted the nomenclature HH6 (and HH6a, HH6b, and HH6s) to acknowledge the previous discoveries and molecular characterizations of the HH1, HH3, HH4, and HH5 embryonic lethal mutations. The HH6 haplotype and HH6 mutation studied in this article should not be confused with the haplotype provisionally named HH6 in Fritz et al. (2013), which was actually associated with the mutation causing complex vertebral malformation. 


\section{Estimation of the Genetic Contribution of MOUNTAIN to the Population Studied}

The genetic contribution of BIS-MAY S-E-L MOUNTAIN-ET (HOLUSAM000002070579) to the genotyped population was estimated using Pedig software version 5 (Boichard, 2002). The pedigree of the 254,075 individuals analyzed in this study was traced in the French Holstein pedigree file using the "ped_util" module. The average number of complete generations traced was 9.9 and the complete pedigree file included 1,015,244 trios. Subsequently, the "prob_orig" module was used for computing MOUNTAIN's contribution.

\section{Fine-Mapping of the HH6 Locus Using Recombinant Haplotypes}

In line with previous studies (Sonstegard et al., 2013; Adams et al., 2016; Michot et al., 2017), we used recombination events among the inbred descendants of the founder bull MOUNTAIN (HOLUSAM000002070579) to fine-map the HH6 locus. More precisely, we screened this sub-population for carriers of both the original lethal haplotype and a recombining haplotype. Such animals cannot be homozygous for the embryonic lethal mutation assuming that it is fully penetrant, which enabled us to exclude from the location interval the identical-by-descent segment for which they are homozygous.

As described in Michot et al. (2017), we defined 2 regions of 40 markers denoted hapL and hapR, located $3 \mathrm{Mb}$, respectively, upstream and downstream of the HH6 haplotype; then, we identified the hapL and hapR source haplotypes of MOUNTAIN. Among its genotyped descendants, we retrieved all the individuals homozygous for HH6_hapL. Then, we extended the HH6_hapL haplotype one SNP at a time toward HH6. Individuals carrying a haplotype different from the initial one (and therefore recombinant) were eliminated. The process was continued until no individual was retained. The same approach was repeated for hapR. On each side, the last remaining individuals defined the left or right boundary of the shortest interval carrying the lethal variant. The refined haplotype contained in the 2 critical intervals was denoted HH6s.

\section{Mining of Whole-Genome Sequencing Data}

In this study, we analyzed the whole-genome sequencing (WGS) data from 296 Holstein bulls from run6 of the 1000 Bull Genomes Consortium (Daetwyler et al., 2014), consisting of 12 heterozygous carriers and 284 noncarriers of the HH6 haplotype (see Supplemental File S1; https://doi.org/10.3168/jds.2017-14119). De- tails on the informatics pipeline used to obtain the list of variants and genotypes for these animals are presented in Daetwyler et al. (2014). Briefly, the genomes of these 296 animals and of 2,037 additional animals belonging to run6 of the 1000 Bull Genomes Consortium were resequenced with Illumina paired-end or SOLiD sequencing technologies. Fastq files were aligned to the reference bovine genome assembly UMD3.1 (Zimin et al., 2009) using BWA version 0.5.9 ( $\mathrm{Li}$ and Durbin, 2009). Single nucleotide polymorphisms and small Indels (insertion/deletions) were called using the mpileup option of SAMtools version 0.1.18 ( $\mathrm{Li}$ et al., 2009). Phred score genotype probabilities were converted into true probabilities, and Beagle version 4.0 (Browning and Browning, 2009) was used to correct genotype calls. Variants located within the critical interval (Chr16: 29,211,284-30,333,319 bp) were annotated using Ensembl Variant Effect Predictor pipeline (VeP) on the Ensembl v88 transcript set (McLaren et al., 2010). The effect of nonsynonymous coding variants was predicted with the SIFT software (Kumar et al., 2009). Finally, the correlation between genotypes derived from WGS and genotypes for the HH6 locus based on haplotype tests was calculated.

In addition, we performed bioinformatics detection of large genomic variations on 5 heterozygous carriers and 50 noncarriers of HH6 haplotype among the 296 animals previously mentioned (see Supplemental Table S1; https://doi.org/10.3168/jds.2017-14119). We performed multi-sample variant calling using Pindel software version 0.2.4y (Ye et al., 2009) and visual detection of structural variants in the 5 heterozygous carriers using the Integrative Genomics Viewer version 2.4 (Robinson et al., 2011).

\section{Large-Scale Genotyping of Candidate Polymorphisms}

The candidate polymorphisms for HH6 g.29773628A $>$ G on chromosome 16 (rs434666183) were included in 2 consecutive custom designs of the Illumina EuroG10K custom SNP BeadChip. At the time of the study, genotype information was available for 46,250 cattle from 12 breeds, including 28,947 Holstein animals (see Results).

\section{Evaluation of the Effect of HH6 on Fertility}

The effect of HH6 haplotype was tested on 2 fertility traits: conception rate and nonreturn rate at $56 \mathrm{~d}$ (NRR56); NRR56 was coded 0 if a second insemination was observed less than $56 \mathrm{~d}$ after the first insemination, and 1 otherwise. As described in Michot et al. (2017), assuming that HH6 causes recessive embryonic 
lethality, the expected decrease in fertility in mating at risk is $0.25 \times \mu$ between 2 carriers (with $\mu$ being the average conception rate or the average NRR56), and $0.25 \frac{1}{2-f} \mu$ between a carrier bull and the daughter of a carrier (where $0.5 \frac{1}{2-f}$ is the proportion of carriers among daughters of carrier bulls and of dams of unknown genotype), and $f$ is the frequency of the deleterious allele. The model used to analyze conception rate and NRR56 was the same as that in Fritz et al. (2013). It accounted for several environmental effects, the fixed effect of the combination of the genotypes of the mated bull and the sire of the cow for HH6 locus, and the random effects of the sire and maternal grandsire of the embryo. Tests were carried out separately for lactating cows and heifers. Finally, a $t$-test was used to determine any significant difference in conception rate and NRR56 between mating at-risk and control groups.

\section{Analysis of RNA Sequencing Data}

Milk cells were collected from 112 lactating Holstein cows and 29 Jersey cows with approval from the Department of Economic Development, Jobs, Transport and Resources (Australia) Animal Ethics Committee (applications 2013-14 and 2014-04). Then, RNA was extracted using Trizol Plus RNA Purification Kit (Ambion/Thermo Fisher Scientific, Waltham, MA) according to the manufacturer's instructions. All RNA samples had RNA integrity numbers $>6$. RNA sequencing (RNASeq) libraries were prepared using the SureSelect Strand Specific RNA Library Prep Kit (Agilent Technologies, Palo Alto, CA) according to the manufacturer's instructions. Each library was uniquely barcoded, randomly assigned to 1 of 4 pools, and sequenced on a HiSeq 3000 (Illumina Inc.) in a 150-cycle paired-end run. Sequence quality was assessed using FastQC version 0.11.4 (https://www.bioinformatics .babraham.ac.uk/projects/fastqc/). QualityTrim (https://bitbucket.org/arobinson/qualitytrim) was used to trim and filter poor quality bases and sequence reads. Adaptor sequences and bases with quality score $<20$ were trimmed from the end of reads. Reads with mean quality score $<20$, or $>3 \mathrm{~N}$ (unknown nucleotide), or more than 3 consecutive bases had quality score $<15$, or final length $<50 \mathrm{bp}$ were discarded. Paired reads were aligned to the Ensembl UMD3.1 bovine genome assembly (https://www.ensembl.org/Bos_taurus/Info/ Index) using TopHat2 version 2.1.1 (Kim et al., 2013) allowing for 2 mismatches. Libraries with $>25$ million read pairs (after quality control filtering) also having $>80 \%$ mapping rate were retained for gene count matrix generation. Gene and exon counts for SDE2 were created using HTSeq version 0.6.1 (Anders et al., 2015). Counts were combined to form a gene/exon by sample count matrix and normalized to take into account library size using DESeq version 1.24.0 (Anders and Huber, 2010). The mpileup option of SaMtools version 0.1.18 ( $\mathrm{Li}$ et al., 2009) was used to count reference and alternate alleles at position g.29773628 on chromosome 16 for all animals.

\section{DNA and RNA Extraction}

Venous blood from 6 SDE2 +/- and 6 SDE2 +/+ cows based on Illumina EuroG10K SNP Chip genotyping data was collected in EDTA tubes and PAXgene Blood RNA Tubes (PreAnalytiX, Hombrechtikon, Switzerland). The tubes were inverted 10 times and stored at $-20^{\circ} \mathrm{C}$. Then, DNA was extracted using the Wizard Genomic DNA Purification Kit (Promega, Charbonnières-les-Bains, France); RNA was extracted using the PAXgene Blood RNA kit (Qiagen, Courtaboeuf, France) according to the manufacturer's recommendations. After extraction, DNA and RNA concentrations were measured using a NanoDrop ND-1000 spectrophotometer (Thermo Fisher Scientific, Villebonsur-Yvette, France) and RNA quality was controlled using a Bionanalyzer 2100 Instrument (Agilent, Les Ulis, France).

\section{Genotyping by PCR and Sanger Sequencing}

Genotyping for candidate variant rs434666183 was performed by PCR and Sanger sequencing using the primers listed in Table 1. The PCR amplification was performed using the Go-Taq Flexi DNA Polymerase (Promega) according to the manufacturer's instructions on a Mastercycler Pro thermocycler (Eppendorf, Montesson, France). The PCR products were subsequently purified and bidirectionally sequenced by Eurofins MWG (Hilden, Germany) using conventional Sanger sequencing. Polymorphisms were detected with the novoSNP software (Weckx et al., 2005).

\section{Analysis of SDE2 Gene Expression}

cDNA samples were prepared using the Superscript III First Strand cDNA Synthesis System (Life Technologies/Thermo Fisher Scientific), with $500 \mathrm{ng}$ of total RNA as starting material, and an equimolar mixture of oligodT and random hexamer primers. Finally, cDNA concentration was measured using a NanoDrop ND1000 spectrophotometer. The primers used for quantitative PCR are listed in Table 1. Primer efficiency and specificity were evaluated on bovine genomic DNA. Quantitative PCR was performed in triplicate with 20 
Table 1. List of primers used in this study

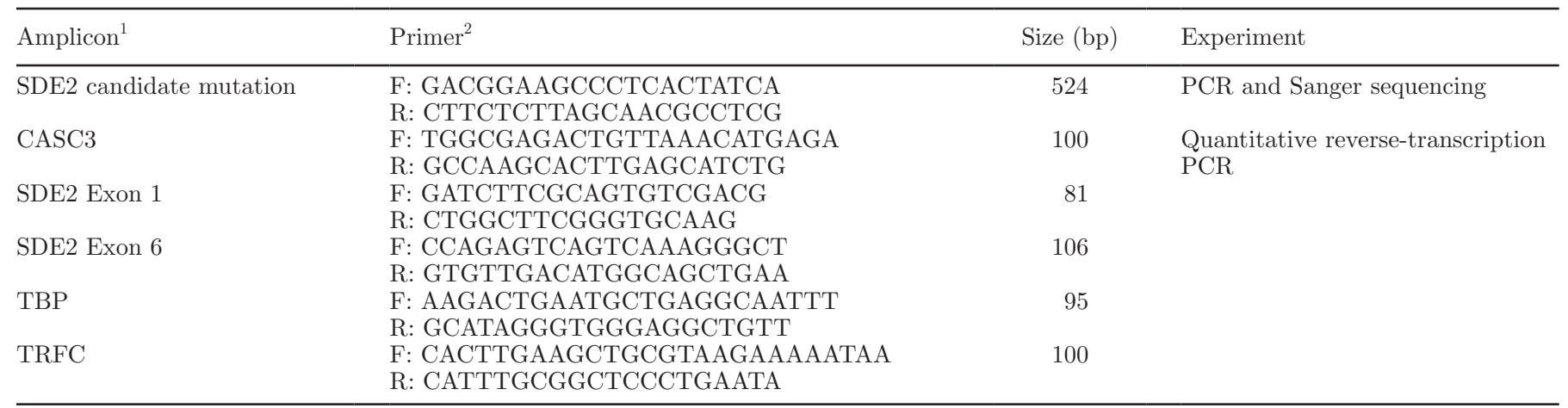

${ }^{1}$ SDE2 $=$ SDE2 telomere maintenance homolog; CASC3 = cancer susceptibility 3 ; TBP $=$ TATA-box binding protein; TRFC $=$ transferrin receptor.

${ }^{2} \mathrm{~F}=$ forward; $\mathrm{R}=$ reverse.

ng of cDNA using the Absolute Blue SYBR Green ROX mix (Thermo Fisher Scientific, Illkirch, France) and the StepOnePlus Real-Time PCR System (Applied Biosystems/Thermo Fisher Scientific). Results were analyzed with the Qbase software using 3 appropriate normalizing genes (transferrin receptor, TRFC; CASC3; and TATA-box binding protein, TBP).

\section{RESULTS AND DISCUSSION}

\section{Screening for Depletion in Homozygous Genotypes in French Holstein}

Analyzing phased genotype data for 254,075 animals and 43,582 SNP using sliding windows of 20 markers, we identified 6 regions with significant depletion in homozygous genotypes (Bonferroni-adjusted $\chi^{2}$ test $P$ value $<0.01$ ) and $\mathrm{N}_{\text {obs }} / \mathrm{N}_{\text {exp }}$ ratios $<0.25$ (Table 2).

Five of these regions corresponded to previously identified recessive mutations causing embryonic lethality (HH1, HH3, HH4, and HH5; Fritz et al., 2013; McClure et al., 2014; Daetwyler et al., 2014; Adams et al., 2016; Schütz et al., 2016) or both embryonic lethality and stillbirth (HBY: Brachyspina; Charlier et al., 2012). For each, we identified a small number of animals that were predicted to be homozygous mutants based on haplotype information. Nevertheless, analysis of EuroG10K SNP BeadChip genotyping data for 9 homozygous animals ( 1 for HBY, 1 for HH1, 2 for HH3, 3 for HH4, and 2 for HH5) revealed that they were all heterozygous for the respective causative mutations and probably carried a recombining haplotype or a copy of the ancestral version of the haplotype predating the mutation event. The HH2 haplotype was not detected with the filter applied in this study, probably because of its very low frequency in the French Holstein population $(0.8 \%)$ and because of incomplete linkage between the haplotype and the causative mutation. Indeed, we observed 4 animals, including 3 lactating cows, that were homozygous for the haplotype described by VanRaden et al. (2011), whereas 16 were expected in our data set according to the Hardy-Weinberg equilibrium equation.

Interestingly, we also detected a new region on chromosome 16 consisting of 2 haplotypes located in close proximity: HH6a (25 consecutive markers from position $27,833,776$ to $29,661,958 \mathrm{bp}$ ) and HH6b (21 consecutive markers from position $31,162,715$ to $32,019,139 \mathrm{bp})$. In our data set, we observed only 3 animals homozygous

Table 2. List of regions displaying significant depletions in homozygotes among 254,075 Holstein cattle ${ }^{1}$

\begin{tabular}{llccccc}
\hline Haplotype & BTA & $\begin{array}{c}\text { Interval } \\
(\mathrm{Mb})\end{array}$ & $\begin{array}{c}\text { Frequency } \\
(\%)\end{array}$ & $\mathrm{N}_{\exp }$ & $\mathrm{N}_{\text {obs }}$ & $\begin{array}{c}\text { Bonferroni-adjusted } \\
\chi^{2} \text { test }\end{array}$ \\
\hline HH3 & 8 & $94.5-95.6$ & 3.1 & 332 & 3 & $7.4 \times 10^{-91}$ \\
HH4 & 1 & $0.1-1.4$ & 4.4 & 301 & 8 & $5.9 \times 10^{-82}$ \\
HBY & 21 & $20.0-21.2$ & 2.7 & 124 & 1 & $6.2 \times 10^{-30}$ \\
HH5 & 9 & $94.8-96.4$ & 1.9 & 117 & 8 & $3.6 \times 10^{-28}$ \\
HH1 & 5 & $63.0-65.6$ & 1.7 & 57 & 1 & $1.1 \times 10^{-11}$ \\
HH6a & 16 & $27.8-29.7$ & 1.2 & 33 & 3 & $2.7 \times 10^{-3}$ \\
HH6b & 16 & $31.2-32.0$ & 1.4 & 43 & 3 & $2.6 \times 10^{-6}$
\end{tabular}

${ }^{1} \mathrm{~N}_{\mathrm{exp}}$ and $\mathrm{N}_{\mathrm{obs}}=$ number of homozygotes expected and observed; only regions with a Bonferroni adjusted $\chi^{2}$ test $P$-value $<0.01$ and a $\mathrm{N}_{\mathrm{obs}} / \mathrm{N}_{\exp }$ ratio $<0.25$ are displayed. 
Table 3. Estimation of losses in conception rate (CR) and nonreturn rate at $56 \mathrm{~d}$ (NRR56) in matings at risk for haplotype $\mathrm{HH}^{1}$

\begin{tabular}{llccc}
\hline $\begin{array}{l}\text { Haplotype } \\
\text { and trait }\end{array}$ & Category & $\begin{array}{c}\text { Matings at } \\
\text { risk (no.) }\end{array}$ & $\begin{array}{c}\text { Proportion of matings at risk compared } \\
\text { with all matings (\%) }\end{array}$ & $\begin{array}{c}\text { Loss } \\
(\%)\end{array}$ \\
\hline HH6 CR & Heifer & 9,471 & 0.08 & -7.92 \\
& Cow & 44,765 & 0.15 & -3.27 \\
HH6 NRR56 & Heifer & 5,398 & 0.07 & -4.70 \\
& Cow & 21,669 & 0.14 & -3.63 \\
\hline
\end{tabular}

${ }^{1}$ Only matings at risk between carrier bulls and daughters of carrier bulls are considered. All losses in CR and NRR56 in at risk versus control matings are significant at $P<0.01$ ( $t$-test).

for HH6a and 3 distinct animals homozygous for HH6b, whereas 33 and 43 homozygous animals, respectively, were expected under neutrality. Haplotype frequencies were $1.2 \%$ for HH6a, $1.4 \%$ for HH6b, and $1.1 \%$ for the chromosomal segment carrying both HH6a and HH6b (denoted HH6 hereafter). Interestingly, no animal was homozygous for HH6, whereas 31 were expected (Bonferroni-adjusted $\chi^{2}$ test, $P=1.7 \times 10^{-4}$ ), suggesting that the mutation associated with HH6 is embryonic lethal.

\section{Analysis of Conception and NRR56 Suggests that HH6/HH6 Embryos Die Before 35 Days of Gestation}

To confirm this expectation and narrow down the stage at which the death of homozygous embryos occurs, we tested the effect of HH6 on conception rate and NRR56 (Table 3). Compared with the control group, we observed a significant loss in these 2 fertility traits for both heifers and adult cows in mating at risk between HH6 carrier bulls and daughters of HH6 carriers $(P<0.05)$. Estimated values were close to the expected effect under the assumption of complete lethality in homozygous embryos $(-6.25$ and $-5.00 \%$, respectively, with an average conception rate of $50 \%$ in heifers and $40 \%$ in cows). Considering that the reproductive cycle averages $21 \mathrm{~d}$ in females, these results indicate that most $\mathrm{HH}^{-/-}$embryos die in the first $35 \mathrm{~d}$ of gestation.

\section{Pedigree Analysis and Fine-Mapping of the HH6 Causal Mutation in Descendants of MOUNTAIN}

Pedigree analysis revealed that the AI bull MOUNTAIN (HOLUSAM000002070579) was the most ancient carrier of the HH6 haplotype among the animals genotyped and the main source for HH6 haplotype in the French population. Indeed, at $2.1 \%$, his genetic contribution to the population of 254,075 animals studied corresponded to approximately twice the frequency of HH6.
Thus, to fine-map the HH6 causal mutation, we screened our population for animals carrying both the original lethal haplotype and a recombining haplotype among the inbred descendants of MOUNTAIN (Figure 1). Such animals cannot be homozygous for the lethal mutation and thus provide useful information. In doing so, we identified several recombining haplotypes, of which 2 enabled us to reduce the critical interval to a region of $1.1 \mathrm{Mb}$ (between positions 29,211,284 and $30,333,319 \mathrm{bp}$ on chromosome 16 on the UMD3.1 assembly).

\section{Screening of WGS Data Identifies a Candidate Polymorphism in the SDE2 Gene}

To identify the causative mutation, we analyzed the WGS data from 296 Holstein bulls (12 HH6 carriers and 284 noncarriers) available from the 1000 Bull Genomes project and calculated the correlation between their genotypes for the HH6 haplotype and for small variants (i.e., SNP and small indels) located in the 1.1-Mb critical interval. The variant showing by far the highest correlation score (0.91; no other variant exceeded a score of 0.5 , Figure 2) was an A-to-G transition at position $29,773,628$ bp on chromosome 16 (g.29773628A > G; rs434666183). Interestingly, this mutation was predicted to affect the initiator codon of SDE2 (the gene encoding SDE2 telomere maintenance homolog). Of note, we did not identify any additional mutation with a correlation score $>0.5$ after detecting large genomic variations on 5 heterozygous carriers and 50 noncarriers of HH6 haplotype.

\section{Large-Scale Genotyping Supports the Causality of the SDE2 g.29773628G Allele}

For further verification, we genotyped the g.29773628A > G variant of SDE2 in a large population of 28,947 Holstein cattle using the Illumina EuroG10K BeadChip. The frequencies of HH6 haplotype and allele g.29773628G were 1.09 and $1.33 \%$, respectively. The 


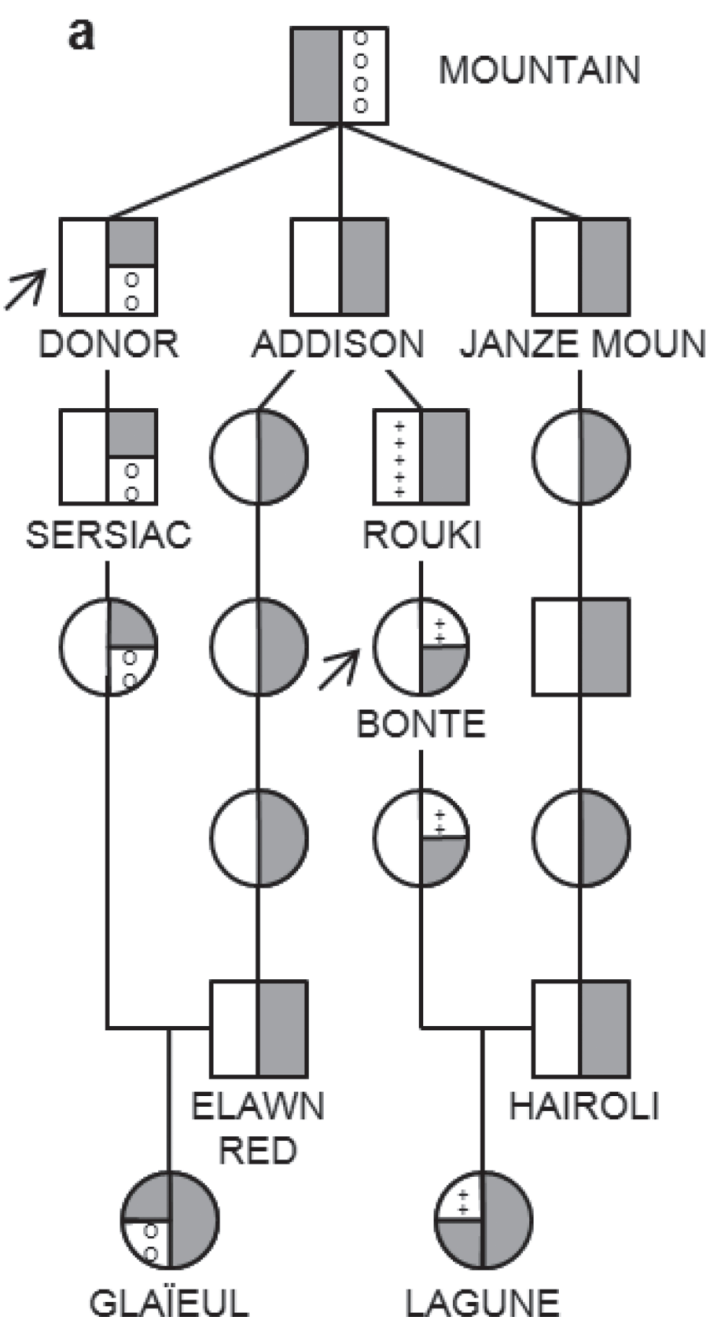

b

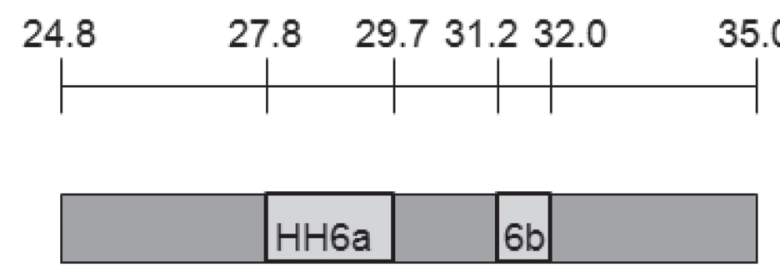

\section{Position (Mb) \\ MOUNTAIN \\ Maternal \\ haplotype \\ MOUNTAIN \\ Paternal \\ haplotype}

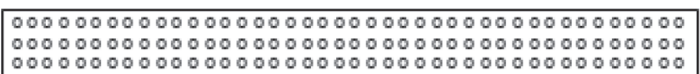
000000000000000000000000000000000000000000

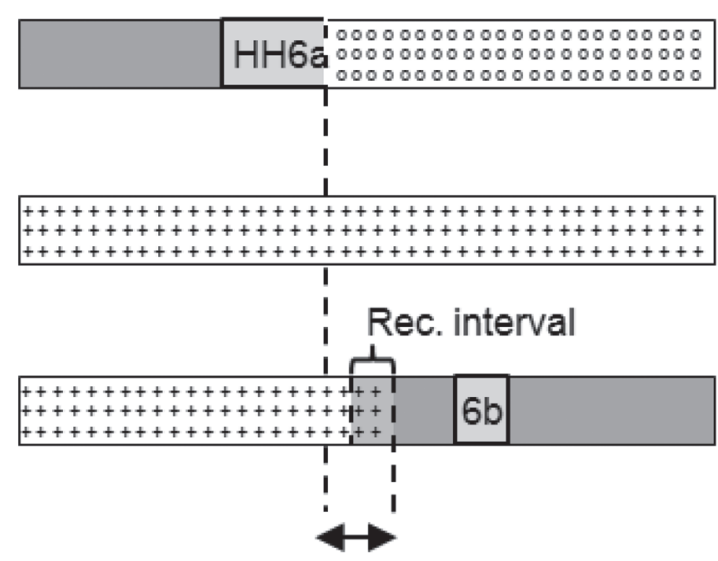

DONOR

Paternal

haplotype

ROUKI

Maternal

haplotype

BONTE

Maternal

haplotype

Figure 1. Fine-mapping of the HH6 locus among the descendants of MOUNTAIN. (a) Pedigree of 2 inbred descendants of MOUNTAIN that enabled the fine-mapping of the HH6-associated embryonic lethal mutation. These 2 animals carry the ancestral chromosomal segment associated with HH6 (depicted in gray) and 2 recombined chromosomes (in white with "O" and "+" marks). Arrows indicate the first carriers of these recombined chromosomes based on Illumina BovineSNP50 (Illumina Inc., San Diego, CA) genotyping data. (b) Details of the original and recombined chromosomes segregating in this pedigree. This diagram presents the positions of the HH6a and HH6b haplotype blocks, of the recombination point or recombination interval (rec. Interval), and of the critical interval for the HH6 locus. The international identifiers of the animals cited in this figure are HOLUSAM000002070579 (MOUNTAIN), HOLAUSM000A00009209 (DONOR), HOLNLDM000839380546 (ADDISON), HOLFRAM003594000491 (JANZE MOUN), HOLFRAM003531174368 (SERSIAC), HOLFRAM002921632624 (ROUKI), HOLFRAF007272390626 (BONTE), HOLFRAM004004223150 (ELAWN RED), HOLFRAM004432722684 (HAIROLI), HOLFRAF004451941329 (GLAIEUL) and HOLFRAF007272390907 (LAGUNE).

HH6 haplotype was found to be always associated with the g.29773628G allele, whereas this allele showed a strong but incomplete association with the HH6 haplotype (Table 4) because of recombinations between the HH6 haplotype and other haplotypes. No homozygous mutant was observed, although 5 were expected in this data set, further supporting that allele g.29773628G is in complete linkage disequilibrium with the embryonic lethal mutation. In addition, analysis of genotype data from 17,303 animals from 11 breeds (Table 5) revealed the absence of the g.29773628G allele outside the Holstein breed, except for 2 female carriers (1 Montbéliarde and 1 Vosgienne) that were found to have Holstein ancestry.

\section{SDE2 Gene Expression Analysis and Prediction of the Consequences of the g.29773628G Allele at the Protein Level}

To investigate putative effects of allele g.29773628G at the mRNA level, we analyzed milk RNA Sequencing data from one heterozygous carrier of the mutation and 91 controls did not show any difference in splicing of SDE2 mRNA between the 2 genotypes (Figure 3 ). 
a

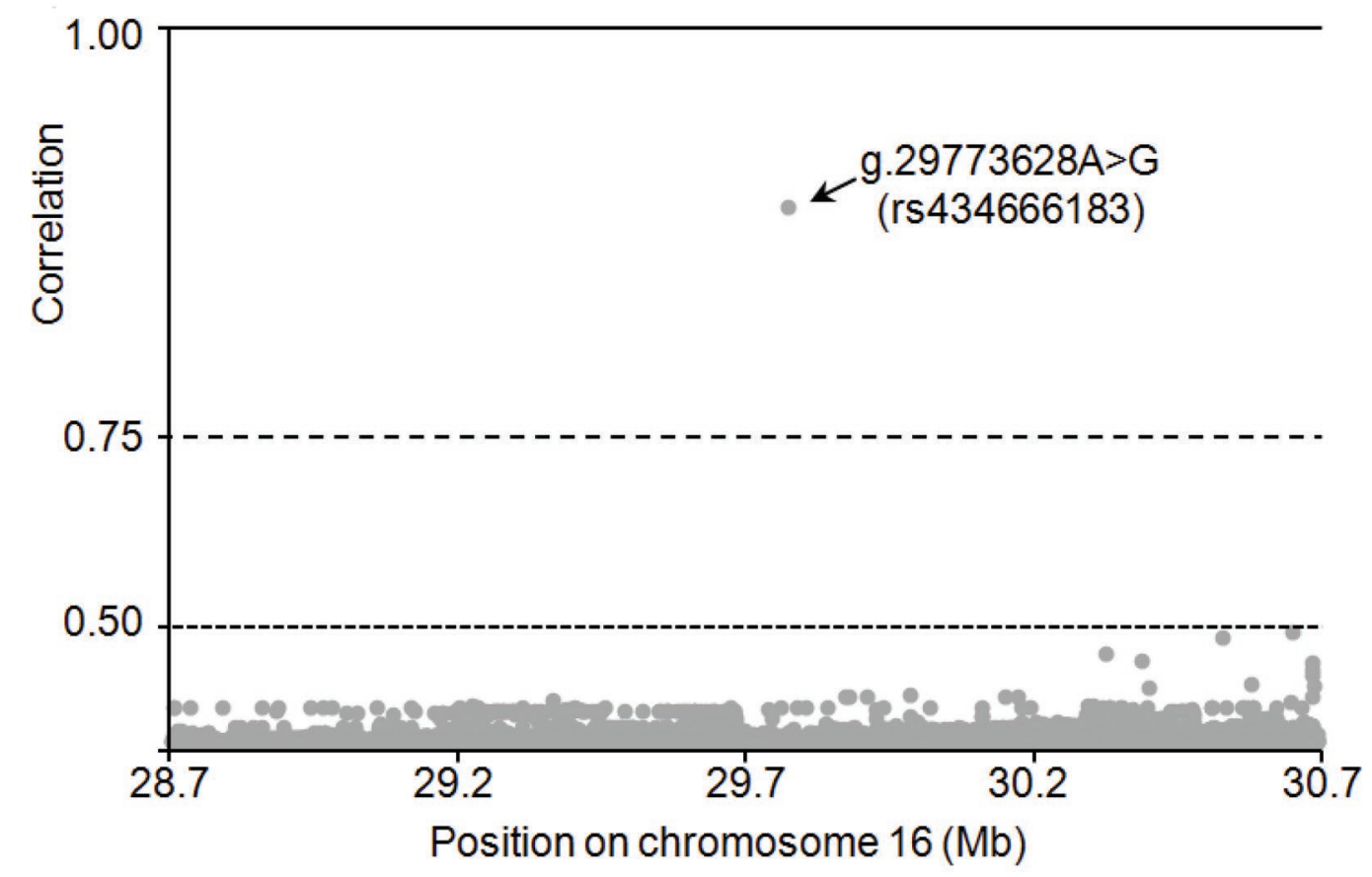

b
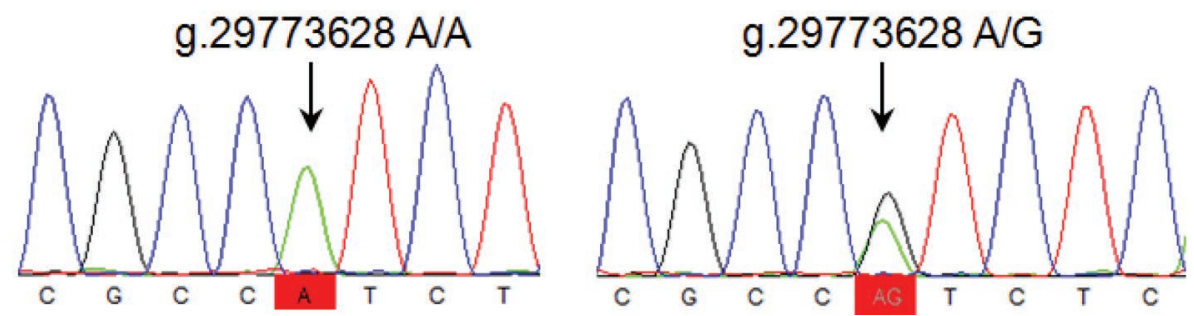

Figure 2. Identification of an initiator codon mutation in SDE2 associated with the HH6 embryonic lethal haplotype. (a) Correlation between genotypes for the HH6 locus based on haplotype tests and genotypes for polymorphisms located in the 1.1-Mb critical interval on chromosome 16. The transformation $10 \times e^{\left[\left(\mathrm{r}^{2}\right)-1\right]}$, where $\mathrm{r}$ is the correlation, was used for graphical representation. The arrow indicates the candidate mutation g.29773628A > G (rs434666183), which is predicted to affect the initiator codon of SDE2. (b) Electrophoregrams from a wild-type animal (A/A left) and a heterozygous carrier (A/G, right) of the g.29773628A $>\mathrm{G}$ substitution. Color version available online.

Interestingly, in the heterozygous carrier, we observed a difference in read count between the 2 alleles of the g.29773628A $>$ G variant (with $16 \mathrm{~A}$ and $5 \mathrm{G}$ ). We thus studied SDE2 expression levels in blood samples from 6

Table 4. Association between HH6 status and genotypes for the g.29773628A > G substitution in 28,947 Holstein cattle $^{1}$

\begin{tabular}{lrccr}
\hline Genotype & $+/+$ & HH6/ & HH6/HH6 & Total \\
\hline g.29773628 A/A & 28,175 & & 0 & 28,175 \\
g.29773628 A/G & 143 & 629 & 0 & 772 \\
g.29773628 G/G & 0 & 0 & 0 & 0 \\
Total & 28,318 & 629 & 0 & 28,947 \\
\hline
\end{tabular}

$\overline{1}+/+=$ noncarriers; HH6/+ and HH6/HH6 = heterozygous and homozygous carriers, respectively, of the HH6 haplotype. heterozygous carriers and 6 control heifers using quantitative PCR. We found a suggestive but not significant reduction of SDE2 expression in 2 independent analyses (Figure 3). Taken together, these results indicate that allele g.29773628G does not significantly alter SDE2 expression and splicing, even if we cannot reject the possibility that a small fraction of mRNA containing the mutant allele might be degraded through a decay mechanism.

As previously mentioned, allele g.29773628G changes the initiator ATG (Met) codon to ACG. Initiation of translation at the closest in-frame Met codon would truncate SDE2 by 83 amino acids, including an 8-amino acid motif conserved among eukaryotes (Figure 4). This perfect conservation reveals a strong 
Table 5. Genotypes of 46,250 animals from 12 breeds for the candidate variant g.29773628A $>$ G on BTA16 according to EuroG10K SNP BeadChip (Illumina Inc., San Diego, CA) genotyping data ${ }^{1}$

\begin{tabular}{lrrc}
\hline Breed & AA & AG & GG \\
\hline Abondance & 474 & 0 & 0 \\
Blonde d'Aquitaine & 153 & 0 & 0 \\
Brown Swiss & 210 & 0 & 0 \\
Charolaise & 655 & 0 & 0 \\
Holstein & 28,175 & 772 & 0 \\
Jersey & 59 & 0 & 0 \\
Limousine & 169 & 0 & 0 \\
Montbéliarde & 12,476 & 1 & 0 \\
Normande & 2,163 & 0 & 0 \\
Parthenaise & 40 & 0 & 0 \\
Tarentaise & 329 & 0 & 0 \\
Vosgienne & 573 & 1 & 0 \\
\hline
\end{tabular}

${ }^{1}$ Pedigree analyses revealed that the Montbéliarde (HOLFRAF006868612733) and Vosgienne (HOLFRAF008843824460) female carriers were not purebred and had 9.4 and $25.0 \%$ Holstein ancestry, respectively. evolutionary constraint and provides evidence that these residues play an important role in the stability or activity of the protein. The SDE2 protein was first identified as a nuclear protein essential for telomeric silencing and genomic stability in Schizosaccharomyces pombe (Sugioka-Sugiyama and Sugiyama, 2011). In fission yeast, SDE2-deficient cells are highly sensitive to several stresses, show a higher frequency of mitotic minichromosome loss, and often form asci that contain spores in abnormal numbers, shapes, or both. Recently, Jo et al. (2016) reported that SDE2 helps cells relieve replication stress and ensures a complete DNA replication process in cultured human HeLa cells; and Thakran et al. (2018) demonstrated that SDE2 supports splicing of selected pre-mRNAs in an intronspecific manner in $S$. pombe. Both teams of researchers also showed that, in fission yeast and humans, SDE2 is

a

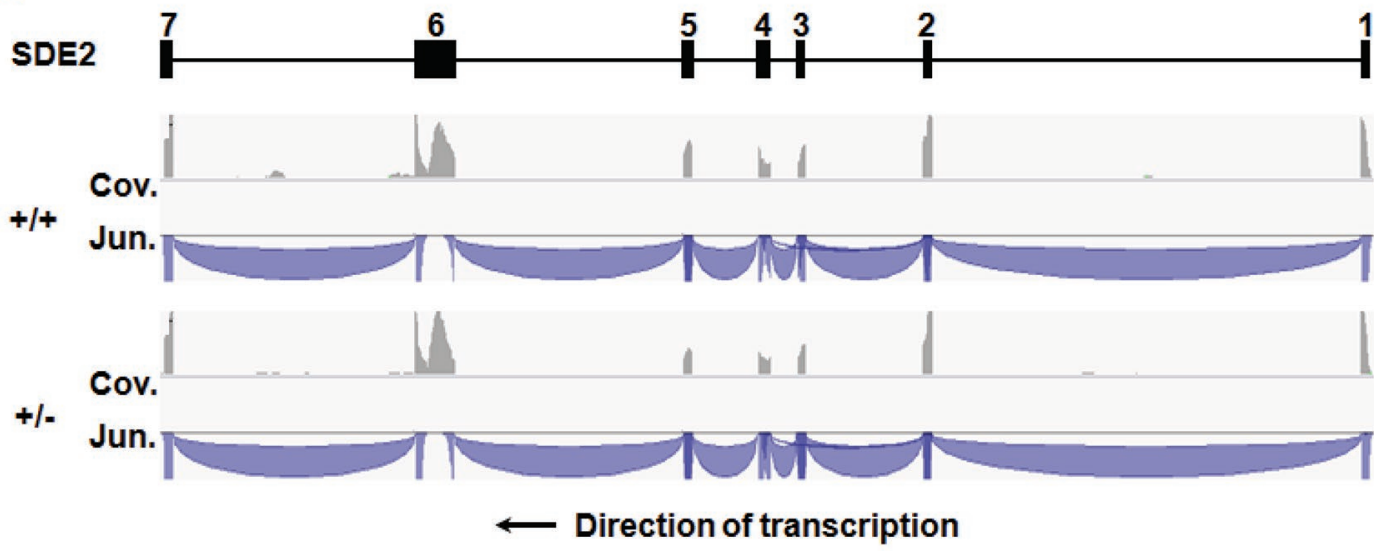

b

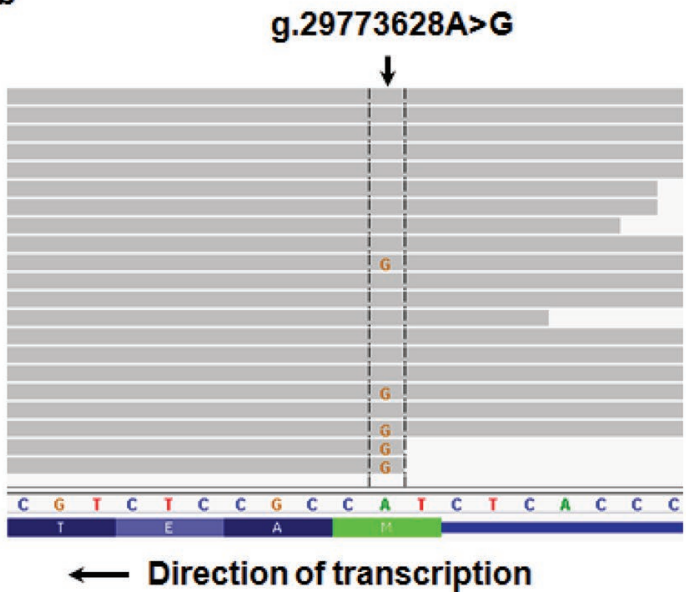

C

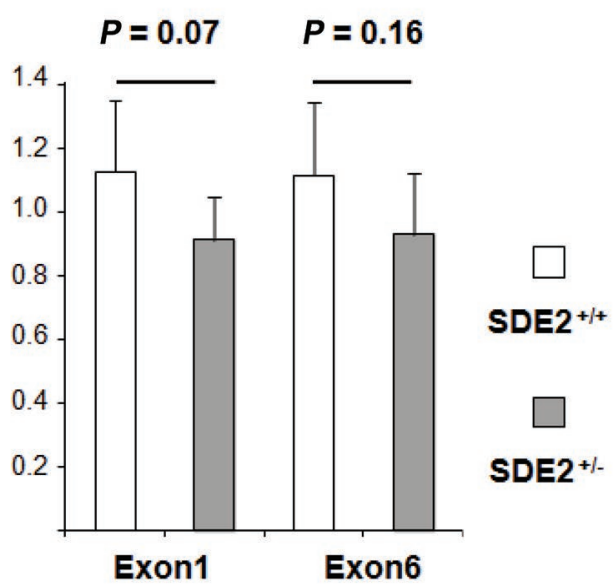

Figure 3. Analysis of SDE2 expression in heterozygous carriers of the initiator codon mutation and wild-type controls. (a) Integrative Genomics Viewer version 2.4 (Robinson et al., 2011) screenshots showing the read coverage (Cov.) and splice junctions (Jun.) in milk RNA sequencing data from a noncarrier $(+/+)$ and a heterozygous carrier $(+/-)$ of the $g .29773628 \mathrm{~A}>\mathrm{G}$ substitution affecting the initiator codon of SDE2. The numbers and positions of SDE2 exons 1 to 7 are presented at the top of the panel. (b) Details on the reads encompassing position g.29773628 on chromosome 16 in milk RNA sequencing data from a heterozygous carrier of the mutation. (c) Quantitative reverse-transcription PCR analysis of SDE2 expression in blood samples from 6 noncarriers (SDE2 ${ }^{++}$) and 6 heterozygous carriers $\left(\mathrm{SDE}^{+/-}\right)$of the mutation. Error bars represent SD. Probabilities were calculated using Fisher's test. Color version available online. 
B. taurus

H. sapiens

G. gallus

P. sinensis

$\mathrm{X}$. laevis

D. rerio

D. elegans

C. intestinalis

o. sativa

S. pombe

B. taurus

H. sapiens

G. gallus

P. sinensis

$\mathrm{X}$. laevis

D. rerio

D. elegans

C. intestinalis

o. sativa

S. pombe
-MAETAALVWLRGPGFGCKAVWCGSAPCSIRDFIRRHCEDQVVPVECF FVKCNGSLVNTS -MAEAAALVWIRGPGFGCKAVRCASGRCTVRDFIHRHCQDQNVPVENF FVKCNGALINTS ----MALLVRE PLCSRARLRALP PGGGSVRGLLRDCARELNVPEESLYAKCNGRLAAGG

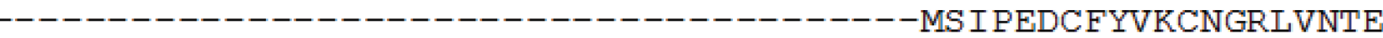
-MASVWVRDALSGRLQLVRVAPGAVALDLLYQEG--TAI PLTDFYVKCNGHLADLE -------MELFVISPDLKFNNLTVAPDSTVSDLIDHFTVKNGVSLTDFYVSSNGRLSRSN ---------MDINI F INNQILINCAS PVNYVELCSQIEKNTNLQPKDYYLVRNGKRLSG----MT SANI LLDLSVIGQGIQHLSI CNDEKIEEKLVRSCVIGENSSFYFTKNGRAFLPG MAEANQYQILVRLLDGRTRCLRFSTTTVSGAALLDAVSALSRVPAASLRLVTGRLDVSPS --MECKTVFLNGDFLKNSVNVNLNRLATVETLLRHVLGDSYETVLERAYLTHQSRIVHPD

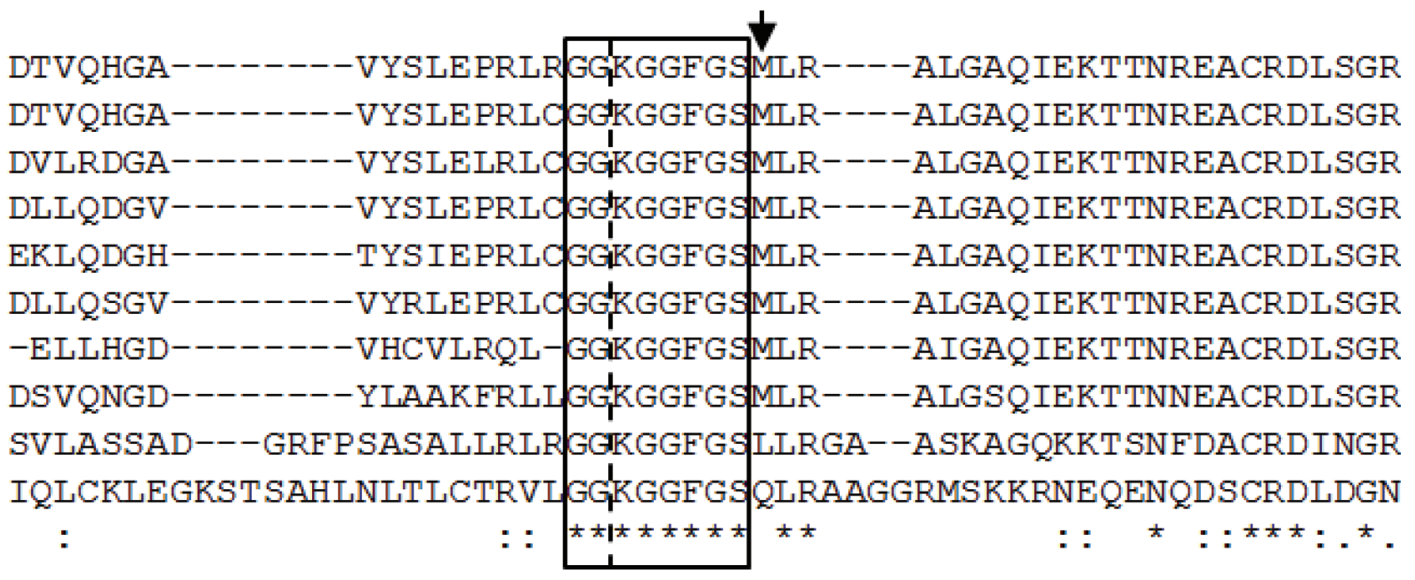

Figure 4. Alignment of the first half of SDE2 telomere maintenance homolog (SDE2) protein sequences from different eukaryotic organisms. Sequence accession numbers in National Center for Biotechnology Information (NCBI; https://www.ncbi.nlm.nih.gov/pubmed/) as follows: NP_001092535.1 (Bos taurus, cow), NP_689821.3 (Homo sapiens, human), XP_015139405.1 (Gallus gallus, chicken), XP_006118111.1 (Pelodiscus sinensis, Chinese softshell turtle), NP_001084858.1 (Xenopus laevis, African clawed frog), NP_001017752.2 (Danio rerio, zebrafish), XP_017113507.1 (Drosophila elegans, fruit fly), XP_002126098.1 (Ciona intestinalis, vase tunicate, sea squirt), XP_015621047.1 (Oryza sativa, rice), and CAB11702.1 (Schizosaccharomyces pombe, fission yeast). The arrow indicates the next in-frame Met codon in the bovine protein after the initiator codon. The black box highlights the 8-AA motif conserved among eukaryotes that would be truncated in the mutant protein. The dashed line indicates the cleavage site of the inactive SDE2 precursor. An asterisk $\left.{ }^{*}\right)$ indicates positions that have a single, fully conserved residue, and : and . indicate conservation between groups of strongly similar and weakly similar properties, respectively. Alignment was performed using ClustalW (Larkin et al., 2007).

translated as an inactive precursor protein harboring an ubiquitin-fold domain linked through an invariant GGKGG motif to a C-terminal domain (referred to as SDE2-C). Precursor processing after the first diglycine motif by ubiquitin-specific proteases generates a shortlived activated SDE2-C fragment with an N-terminal lysine residue. It is worth noting that the cleavage site is part of the conserved 8-amino acid sequence previously mentioned (Figure 4). The absence of this motif in the mutant bovine SDE2 protein is thus predicted to have major consequences for its function. Considering the importance of mitosis in embryo development and the key role played by SDE2-C in maintaining genome stability and ensuring the completion of DNA replication during this process, we might expect that SDE2deficient bovine embryos die after a few cell divisions. This early embryonic mortality is perfectly consistent with a negative effect of HH6 haplotype on NRR56 and with the absence of live homozygous animals for SDE2 allele g.29773628G and HH6 haplotype in the French Holstein population.

\section{CONCLUSIONS}

Combining a genome scan for homozygous haplotype deficiency with whole-genome sequence data analysis is a very effective approach to identify embryonic lethal mutations. The power of this approach depends mainly on the number of genotypes and sequences available. Here, we analyzed the largest panel (to our knowledge) of genotypes and WGS published for this type of study in cattle. We demonstrate that we can map new haplotypes segregating at low frequency $(\sim 1 \%)$ in the population and identify a single mutation in strong association with this haplotype. Using large-scale genotyping, we confirmed the absence of live homozygous animals and 
perfect linkage disequilibrium between the candidate mutation and the embryonic lethal phenotype. Finally, using complementary analyses, we predicted the effect of this mutation affecting the initiator codon of the SDE2 protein. We recommend regular genome scans for homozygous haplotype deficiencies, as genotype data are accumulating, to identify new rare embryonic lethal mutations. The low frequency of these mutations should facilitate their elimination from cattle populations by excluding the rare male carriers from reproduction.

\section{ACKNOWLEDGMENTS}

This study is part of the BOVANO project (ANR14-CE19-0011) funded by the French Agence Nationale de la Recherche (ANR, Paris, France) and APIS-GENE (Paris, France). The authors are grateful to the partners of the 1000 Bull Genomes Consortium (http://www .1000bullgenomes.com/) for the excellent collaboration.

\section{REFERENCES}

Adams, H. A., T. Sonstegard, P. M. VanRaden, D. J. Null, C. P. Van Tassell, D. M. Larkin, and H. A. Lewin. 2016. Identification of a nonsense mutation in APAF1 that is likely causal for a decrease in reproductive efficiency in Holstein dairy cattle. J. Dairy Sci. 99:6693-6701. https://doi.org/10.3168/jds.2015-10517.

Anders, S., and W. Huber. 2010. Differential expression analysis for sequence count data. Genome Biol. 11:R106. https://doi.org/10 .1186/gb-2010-11-10-r106.

Anders, S., P. T. Pyl, and W. Huber. 2015. HTSeq-A Python framework to work with high-throughput sequencing data. Bioinformatics 31:166-169. https://doi.org/10.1093/bioinformatics/btu638.

Boichard, D. 2002. Pedig: A fortran package for pedigree analysis suited to large populations. Paper 28-13 in Proc. 7th World Congress on Genetics Applied to Livestock Production, Montpellier, France. INRA, Castanet-Tolosan, France.

Boichard, D., H. Chung, R. Dassonneville, X. David, A. Eggen, S. Fritz, K. J. Gietzen, B. J. Hayes, C. T. Lawley, T. S. Sonstegard, C. P. Van Tassell, P. M. VanRaden, K. A. Viaud-Martinez, and G. R. Wiggans.Bovine LD Consortium. 2012a. Design of a bovine low-density SNP array optimized for imputation. PLoS One 7:e34130. https://doi.org/10.1371/journal.pone.0034130.

Boichard, D., F. Guillaume, A. Baur, P. Croiseau, M. N. Rossignol, M. Y. Boscher, T. Druet, L. Genestout, J. J. Colleau, L. Journaux, V. Ducrocq, and S. Fritz. 2012b. Genomic selection in French dairy cattle. Anim. Prod. Sci. 52:115-120. https://doi.org/10.1071/ AN11119.

Browning, B. L., and S. R. Browning. 2009. A unified approach to genotype imputation and haplotype-phase inference for large data sets of trios and unrelated individuals. Am. J. Hum. Genet. 84:210-223. https://doi.org/10.1016/j.ajhg.2009.01.005.

Charlier, C., J. S. Agerholm, W. Coppieters, P. Karlskov-Mortensen, W. Li, G. de Jong, C. Fasquelle, L. Karim, S. Cirera, N. Cambisano, N. Ahariz, E. Mullaart, M. Georges, and M. Fredholm. 2012. A deletion in the bovine FANCI gene compromises fertility by causing fetal death and brachyspina. PLoS One 7:e43085. https:// doi.org/10.1371/journal.pone.0043085.

Daetwyler, H. D., A. Capitan, H. Pausch, P. Stothard, R. van Binsbergen, R. F. Brondum, X. Liao, A. Djari, S. C. Rodriguez, C. Grohs, D. Esquerre, O. Bouchez, M. N. Rossignol, C. Klopp, D. Rocha, S. Fritz, A. Eggen, P. J. Bowman, D. Coote, A. J. Chamberlain, C. Anderson, C. P. VanTassell, I. Hulsegge, M. E. Goddard, B. Guld- brandtsen, M. S. Lund, R. F. Veerkamp, D. A. Boichard, R. Fries, and B. J. Hayes. 2014. Whole-genome sequencing of 234 bulls facilitates mapping of monogenic and complex traits in cattle. Nat. Genet. 46:858-865. https://doi.org/10.1038/ng.3034.

Fritz, S., A. Capitan, A. Djari, S. C. Rodriguez, A. Barbat, A. Baur, C. Grohs, B. Weiss, M. Boussaha, D. Esquerre, C. Klopp, D. Rocha, and D. Boichard. 2013. Detection of haplotypes associated with prenatal death in dairy cattle and identification of deleterious mutations in GART, SHBG and SLC37A2. PLoS One 8:e65550. https://doi.org/10.1371/journal.pone.0065550.

Illumina. 2015. Data Sheet: Agrigenomics. BovineHD Genotyping BeadChip. Accessed Apr. 3, 2017. http://www.illumina.com/ documents/products/datasheets/datasheet_bovineHD.pdf.

Jo, U., W. Cai, J. Wang, Y. Kwon, A. D. D'Andrea, and H. Kim. 2016. PCNA-dependent cleavage and degradation of SDE2 regulates response to replication stress. PLoS Genet. 12:e1006465. https://doi .org/10.1371/journal.pgen.1006465.

Kim, D., G. Pertea, C. Trapnell, H. Pimentel, R. Kelley, and S. L. Salzberg. 2013. TopHat2: Accurate alignment of transcriptomes in the presence of insertions, deletions and gene fusions. Genome Biol. 14:R36. https://doi.org/10.1186/gb-2013-14-4-r36.

Kumar, P., S. Henikoff, and P. C. Ng. 2009. Predicting the effects of coding non-synonymous variants on protein function using the SIFT algorithm. Nat. Protoc. 4:1073-1081. https://doi.org/10 $.1038 /$ nprot.2009.86

Larkin, M. A., G. Blackshields, N. P. Brown, R. Chenna, P. A. McGettigan, H. McWilliam, F. Valentin, I. M. Wallace, A. Wilm, R. Lopez, J. D. Thompson, T. J. Gibson, and D. G. Higgins. 2007. Clustal W and Clustal X version 2.0. Bioinformatics 23:2947-2948. https://doi.org/10.1093/bioinformatics/btm404.

Li. H., and R. Durbin. 2009. Fast and accurate short read alignment with Burrows-Wheeler transform. Bioinformatics 25:1754-1760. https://doi.org/10.1093/bioinformatics/btp324.

Li, H., B. Handsaker, A. Wysoker, T. Fennell, J. Ruan, N. Homer, G. Marth, G. Abecasis, R. Durbin, and the 1000 Genome Project Data Processing Subgroup. 2009. The Sequence Alignment/Map format and SAMtools. Bioinformatics 25:2078-2079. https://doi .org/10.1093/bioinformatics/btp352.

Matukumalli, L. K., C. T. Lawley, R. D. Schnabel, J. F. Taylor, M. F Allan, M. P. Heaton, J. O'Connell, S. S. Moore, T. P. L. Smith, T. S. Sonstegard, and C. P. Van Tassell. 2009. Development and characterization of a High Density SNP genotyping assay for cattle. PLoS One 4:e5350. https://doi.org/10.1371/journal.pone.0005350.

McClure, M. C., D. Bickhart, D. Null, P. VanRaden, L. Y. Xu, G. Wiggans, G. Liu, S. Schroeder, J. Glasscock, J. Armstrong, J. B. Cole, C. P. Van Tassell, and T. S. Sonstegard. 2014. Bovine exome sequence analysis and targeted SNP genotyping of recessive fertility defects $\mathrm{BH} 1, \mathrm{HH} 2$, and $\mathrm{HH} 3$ reveal a putative causative mutation in SMC2 for HH3. PLoS One 9:e92769. https://doi.org/ 10.1371/journal.pone.0092769.

McLaren, W., B. Pritchard, D. Rios, Y. Chen, P. Flicek, and F. Cunningham. 2010. Deriving the consequences of genomic variants with the Ensembl API and SNP effect predictor. Bioinformatics 26:2069-2070. https://doi.org/10.1093/bioinformatics/btq330.

Michot, P., S. Fritz, A. Barbat, M. Boussaha, M.-C. Deloche, C. Grohs, C. Hoze, L. Le Berre, D. Le Bourhis, O. Desnoes, P. Salvetti, L. Schibler, D. Boichard, and A. Capitan. 2017. A missense mutation in PFAS (phosphoribosylformylglycinamidine synthase) is likely causal for embryonic lethality associated with the MH1 haplotype in Montbéliarde dairy cattle. J. Dairy Sci. 100:8176-8187. https:// doi.org/10.3168/jds.2017-12579.

Pausch, H., H. Schwarzenbacher, J. Burgstaller, K. Flisikowski, C. Wurmser, S. Jansen, S. Jung, A. Schnieke, T. Wittek, and R. Fries. 2015. Homozygous haplotype deficiency reveals deleterious mutations compromising reproductive and rearing success in cattle. BMC Genomics 16:312. https://doi.org/10.1186/s12864-015-1483 -7 .

Robinson, J. T., H. Thorvaldsdóttir, W. Winckler, M. Guttman, E S. Lander, G. Getz, and J. P. Mesirov. 2011. Integrative genom- 
ics viewer. Nat. Biotechnol. 29:24-26. https://doi.org/10.1038/nbt .1754 .

Sargolzaei, M., J. P. Chesnais, and F. S. Schenkel. 2014. A new approach for efficient genotype imputation using information from relatives. BMC Genomics 15:478. https://doi.org/10.1186/1471 $-2164-15-478$.

Schütz, E., C. Wehrhahn, M. Wanjek, R. Bortfeld, W. E. Wemheuer, J. Beck, and B. Brenig. 2016. The Holstein Friesian lethal haplotype 5 (HH5) results from a complete deletion of TBF1M and cholesterol deficiency $(\mathrm{CDH})$ from an ERV-(LTR) insertion into the coding region of APOB. PLoS One 11:e0154602. https://doi .org/10.1371/journal.pone.0154602.

Schwarzenbacher, H., J. Burgstaller, F. R. Seefried, C. Wurmser, M. Hilbe, S. Jung, C. Fuerst, N. Dinhopl, H. Weissenbock, B. FuerstWaltl, M. Dolezal, R. Winkler, O. Grueter, U. Bleul, T. Wittek, R. Fries, and H. Pausch. 2016. A missense mutation in TUBD1 is associated with high juvenile mortality in Braunvieh and Fleckvieh cattle. BMC Genomics 17:400. https://doi.org/10.1186/s12864 $-016-2742-y$.

Sonstegard, T. S., J. B. Cole, P. M. VanRaden, C. P. Van Tassell, D. J. Null, S. G. Schroeder, D. Bickhart, and M. C. McClure. 2013. Identification of a nonsense mutation in CWC15 associated with decreased reproductive efficiency in Jersey cattle. PLoS One 8:e54872. https://doi.org/10.1371/journal.pone.0054872.

Sugioka-Sugiyama, R., and T. Sugiyama. 2011. Sde2: A novel nuclear protein essential for telomeric silencing and genomic stability in Schizosaccharomyces pombe. Biochem. Biophys. Res. Commun. 406:444-448. https://doi.org/10.1016/j.bbrc.2011.02.068.
Thakran, P., P. A. Pandit, S. Datta, K. K. Kolathur, J. A. Pleiss, and S. K. Mishra. 2018. Sde2 is an intron-specific pre-mRNA splicing regulator activated by ubiquitin-like processing. EMBO J. 37:89101. https://doi.org/10.15252/embj.201796751.

VanRaden, P. M., K. M. Olson, D. J. Null, and J. L. Hutchison. 2011. Harmful recessive effects on fertility detected by absence of homozygous haplotypes. J. Dairy Sci. 94:6153-6161. https://doi.org/10 $.3168 / \mathrm{jds} .2011-4624$.

Venhoranta, H., H. Pausch, K. Flisikowski, C. Wurmser, J. Taponen, H. Rautala, A. Kind, A. Schnieke, R. Fries, H. Lohi, and M. Andersson. 2014. In-frame exon skipping in UBE3B is associated with developmental disorders and increased mortality in cattle. BMC Genomics 15:890. https://doi.org/10.1186/1471-2164-15-890.

Weckx, S., J. Del-Favero, R. Rademakers, L. Claes, M. Cruts, P. De Jonghe, C. Van Broeckhoven, and P. De Rijk. 2005. NovoSNP, a novel computational tool for sequence variation discovery. Genome Res. 15:436-442. https://doi.org/10.1101/gr.2754005.

Ye, K. H. M. Schulz, Q. Long, R. Apweiler, and Z. Ning. 2009. Pindel: A pattern growth approach to detect break points of large deletions and medium sized insertions from paired-end short reads. Bioinformatics 25:2865-2871. https://doi.org/10.1093/ bioinformatics/btp394.

Zimin, A. V., A. L. Delcher, L. Florea, D. R. Kelley, M. C. Schatz, D. Puiu, F. Hanrahan, G. Pertea, C. P. Van Tassell, T. S. Sonstegard, G. Marcais, M. Roberts, P. Subramanian, J. A. Yorke, and S. L. Salzberg. 2009. A whole-genome assembly of the domestic cow, Bos taurus. Genome Biol. 10:R42. https://doi.org/10.1186/ gb-2009-10-4-r42. 